\title{
XLII. On the focal lengths and aberrations of a thin lens of uniaxal crystal, bounded by surfaces which are of revolution about its axis
}

\section{Sir William Rowan Hamilton P.R.I.A.}

To cite this article: Sir William Rowan Hamilton P.R.I.A. (1841) XLII. On the focal lengths and aberrations of a thin lens of uniaxal crystal, bounded by surfaces which are of revolution about its axis, Philosophical Magazine Series 3, 19:124, 289-294, DOI: 10.1080/14786444108650420

To link to this article: http://dx.doi.org/10.1080/14786444108650420

曲 Published online: 01 Jun 2009.

Submit your article to this journal $[\pi$

ЏII Article views: 2

Q View related articles ¿ 
Sir W. R. Hamilton on a Lens of Uniaxal Crystal. 289

$$
\begin{gathered}
\text { Now } \phi=\omega+\phi-\omega, \text { hence } \\
\cos \phi=\cos \omega \cos (\phi-\omega)-\sin \omega \sin (\phi-\omega) \text {, or } \\
\cos ^{2} \phi=\cos ^{2} \omega \cos ^{2}(\phi-\omega)+\sin ^{2} \omega \sin ^{2}(\phi-\omega)-2 \sin \omega \cos \omega \\
\cos (\phi-\omega) \sin (\phi-\omega) \text {. Similarly, } \\
\sin ^{2} \phi=\sin ^{2} \omega \cos ^{2}(\phi-\omega)+\cos ^{2} \omega \sin ^{2}(\phi-\omega)+2 \sin \omega \cos \omega \\
\cos (\phi-\omega) \sin (\phi-\omega) .
\end{gathered}
$$

Putting these values of $\cos ^{2} \phi, \sin ^{2} \phi$ in the first member of (V.) and eliminating, we find

$$
(\mathrm{A}-\mathrm{B}) \sin 2 \omega \sin 2(\phi-\omega)=0 \ldots(\mathrm{VI} .)
$$

Now as $\phi$ is quite arbitrary, we may always assume it differing in value from $\omega$, hence we may consider $\sin 2(\uparrow-\omega)$ as always different from zero; dividing then by this factor, equation (VI.) is thus reduced to

$$
(\mathrm{A}-\mathrm{B}) \sin 2 \omega=0 \ldots \ldots \text { (VII.) }
$$

This equation is satisfied when $A=B$ by any values of $w$, or, in other words, when the moments of inertia round two of the principal axes passing through the centre of gravity are equal; any pair of rectangular axes in a plane perpendicular to the third principal axe are also principal axes.

When $A$ is not equal to $B$, equation (VII.) can only be satisfied by the values $\omega=0$, or $\omega=\frac{\pi}{2}$, or the principal axe $\mathrm{O} x^{\prime}$ coincides with $\mathrm{O} x$, which is parallel to $\mathrm{G} \mathrm{X}$, hence we may deduce the following theorem :-

The principal axes of rotation of a body passing through any point assumed on one of the three principal axes through the centre of gravity, are alivays parallel to these axes.

XLII. On the Focal Lengths and Aberrations of a thin Lens of Uniaxal Crystal, bounded by Surfaces which are of Revolution about its Axis. By Sir William Rowan HamilTon, P.R.I.A., Member of several Scientific Societies at Home and Abroad, Professor of Astronomy in the University of Dublin, and Royal Astronomer of Ireland*.

THE following short investigation may perhaps be not without interest to the students of mathematical optics, as serving to illustrate a general method, and to correct an important error into which an eminent writer has fallen.

1. Let a ray of ordinary light, in vacuo, and in the plane of $x z$, proceeding from or towards a given point on the axis of $z$, be incident nearly perpendicularly on a given surface of revolution, and there undergo extraordinary refraction at en-

- Communicated by the Author.

Phil. Mag. S. 3. Vol. 19. No. 124. Oct. 1841. 
tering a given uniaxal crystal, of which the optical axis coincides with the axis of revolution and of $z$. It is required to determine the intersection of the refracted ray with the axis; the distance of the point of incidence from the vertex, or the semiaperture of the crystal, being given.

2. The law of this extraordinary refraction may (according to the general methods of my 'Theory of Systems of Rays,' published in the Transactions of the Royal Irish Academy,) be thus expressed:

$$
\left(\sigma-\alpha^{\prime}\right) \delta \xi+\left(u-\gamma^{\prime}\right) \delta \zeta=0 . \ldots .
$$

In this formula, $\xi, \zeta$ are (in the plane of $x z$ ) the rectangular coordinates of incidence, and are connected by the equation of the meridional section of the given refracting surface of revolution, which equation we may suppose to be (at least nearly enough for our present purpose) developed under the form

$$
\zeta=\frac{r}{2} \xi^{2}+\frac{s}{4} \xi^{4}, \ldots \ldots \ldots
$$

$r$ being the curvature at the vertex, and $s$ being another constant, which in the case of a spheric surface is half of the cube of that curvature; $\alpha^{\prime}, \gamma^{\prime}$ are the cosines of the inclinations of the incident ray to the positive semiaxes of $x z$, so that they are connected by the relation

$$
\alpha^{12}+\gamma^{12}=1 ; \ldots \ldots \ldots .
$$

and $\sigma, v$ are the components of normal slowness of the extraordinary wave within the crystal, so that, if $\mu$ be the ordinary and $\nu$ the extraordinary index, these components are connected by the relation

$$
\mu^{2} \sigma^{2}+\nu^{2} u^{2}=\mu^{2} \nu^{2} .
$$

And these quantities, $\alpha^{\prime} \gamma^{\prime}$ a $v$, are such, that if $x^{\prime} z^{\prime}$ be the coordinates of any point on the incident ray, we have

$$
\left(\xi-x^{\prime}\right) \delta \alpha^{\prime}+\left(\zeta-z^{\prime}\right) \delta \gamma^{\prime}=0 ; \ldots .
$$

and if $x y$ be the coordinates of any point on the refracted ray, we have

$$
(x-\xi) \delta \sigma+(z-\zeta) \delta v=0 . \ldots \ldots
$$

3. Making then

$$
T=\xi\left(\sigma-\alpha^{\prime}\right)+\zeta\left(u-\gamma^{\prime}\right), \ldots \ldots
$$

we have first the equation

$$
\frac{\delta T}{\delta \xi}=0, \ldots \ldots \ldots \ldots
$$

which contains the law of extraordinary refraction, and by which $\xi$ can be eliminated from the expression of $T$, so as to 
leave that quantity $\mathrm{T}$ expressed as a function of $\alpha^{\prime}$ and $\sigma$ only; $\xi$ being here treated as a known function of $\xi, \gamma^{\prime}$ of $\alpha^{\prime}$, and $v$ of $\sigma$. And since, by (1.) (5.) (6.),

$$
\delta \mathrm{T}=x \delta \sigma+z \delta v-x^{\prime} \delta \alpha^{\prime}-z^{\prime} \delta \gamma^{\prime}, \ldots .
$$

we have, by (3.) and (4.), the following equations, for the incident and refracted rays respectively:

$$
\begin{array}{r}
-x^{\prime}+\frac{\alpha^{\prime}}{\gamma^{\prime}} z^{\prime}=\frac{\delta \mathrm{T}}{\delta \alpha^{\prime}} ; \ldots \ldots \\
x-\frac{\mu^{2} \sigma}{\nu^{2} v} \approx=\frac{\delta \mathrm{T}}{\delta \sigma} . \ldots .
\end{array}
$$

4. The approximate equation (2.) of the section of the refracting surface, which gives $\zeta$ as an explicit function of $\xi$, is now to be combined with the following analogous expressions for the functions $\gamma^{\prime}$ and $v$, deduced from the relations (3.) and (4.):

$$
\gamma^{\prime}=1-\frac{\alpha^{\prime 2}}{2} ; v=\mu-\frac{\mu \sigma^{2}}{2 v^{2}} ; \ldots \ldots
$$

and thus the expression (7.) for $T$ becomes, if we neglect terms which are small of the sixth dimension with respect to $\alpha^{\prime}, \sigma, \xi$ :

$$
\begin{aligned}
& \mathrm{T}=\mathrm{T}^{(2)}+\mathrm{T}^{(4)} ; \ldots \ldots \ldots \\
& \mathrm{T}^{(2)}=\xi\left(\sigma-\alpha^{\prime}\right)+\frac{1}{2} r \xi^{2}(\mu-1) ; \ldots \ldots \\
& \mathrm{T}^{(4)}=\frac{1}{4} s \xi^{4}(\mu-1)-\frac{1}{4} r \xi^{2}\left(\frac{\mu \sigma^{2}}{\nu^{2}}-\alpha^{\prime 2}\right) \ldots
\end{aligned}
$$

And to eliminate $\xi$, it is sufficient to employ the equation (8.) under the approximate form

$$
0=\frac{\delta T(2)}{\delta \xi}=\sigma-\alpha^{\prime}+r \xi(\mu-1) ; \ldots \ldots
$$

for although the complete expression for the abscissa $\xi$ of incidence contains terms of the third and higher dimensions with respect to $\alpha^{\prime}$ and $\sigma$, yet the introduction of these terms of $\xi$ would only introduce terms of the sixth and higher dimensions, in the expression for the function $\mathrm{T}$.

5. Retaining therefore $\xi$ as an auxiliary symbol, of which the meaning is determined by the formula (16.), and making the abscissæ $x^{\prime}$ and $x$ to vanish in the equations of the two rays, (10.) and (11.), in order to discover the relation between the ordinates $z^{\prime}$ and $z$ of the intersections of those two rays with the axis, we find 


$$
\begin{aligned}
& +\alpha^{\prime} z^{\prime}\left(1+\frac{\alpha^{\prime 2}}{2}\right)=-\xi+\frac{\delta T^{(4)}}{\delta \alpha^{\prime}} ; \ldots \\
& -\frac{\mu \sigma z}{y^{2}}\left(1+\frac{\sigma^{2}}{2 \nu^{2}}\right)=+\xi+\frac{\delta T^{(4)}}{\delta \sigma} ; \ldots
\end{aligned}
$$

and therefore, taking the reciprocals,

$$
\begin{gathered}
-\frac{1}{z^{\prime}}=\frac{\alpha^{\prime}}{\xi}\left(1+\frac{\alpha^{\prime 2}}{2}\right)+\frac{\alpha^{\prime}}{\xi^{2}} \frac{\delta \mathrm{T}^{(4)}}{\delta \alpha^{\prime}} ; \ldots \\
\frac{\nu^{2}}{\mu z}=\frac{-\sigma}{\xi}\left(1+\frac{\sigma^{2}}{2 \nu^{2}}\right)+\frac{\sigma}{\xi^{2}} \frac{\partial \mathrm{T}^{(4)}}{\delta \sigma} \ldots
\end{gathered}
$$

Adding these last two equations, attending to the value (16.) of $\xi$, and observing that $T^{(4)}$, after substitution of that value, becomes a homogeneous function of the fourth dimension of $\alpha^{\prime}$ and $\sigma$, so that

$$
\alpha^{\prime} \frac{\delta \mathbf{T}^{(4)}}{\delta \alpha^{\prime}}+\sigma \frac{\delta \mathbf{T}^{(4)}}{\delta \sigma}=4 \mathbf{T}^{(4)}, \ldots \ldots \ldots
$$

we find this relation:

$$
\frac{\nu^{2}}{\mu z}-\frac{1}{z^{\prime}}-(\mu-1) r=\frac{1}{2 \xi}\left(\alpha^{\prime 3}-\frac{\sigma^{3}}{\nu^{2}}\right)+\frac{4}{\xi^{2}} \mathrm{~T}^{(4)} \text {. }
$$

And changing, in the second member, $\alpha^{\prime}$ and $\sigma$ to their approximate values given by (19.) and (20.), namely,

we find

$$
\alpha^{\prime}=-\frac{\xi}{z^{\prime}}, \quad \sigma=-\frac{\nu^{2} \xi}{\mu z}, \ldots \ldots
$$

$$
\begin{aligned}
& \frac{\nu^{2}}{\mu z}-\frac{1}{z^{\prime}}-(\mu-1) r \\
& \quad=\frac{\xi^{2}}{2}\left\{\frac{v^{4}}{\mu^{3} z^{3}}-\frac{1}{z^{13}}+2(\mu-1) s-2 r\left(\frac{\nu^{2}}{\mu z^{2}}-\frac{1}{z^{12}}\right)\right\} .
\end{aligned}
$$

By suppressing the second member, we get the intersection of the axis with a refracted ray infinitely near it, or the extraordinary focus of the central rays; by taking account of that member we get the longitudinal aberration.

6. As a verification, we may consider the case of an ordinary refraction at a spheric surface as being included in the foregoing, and the formula for that case must result from the equation (24.), by making therein

$$
\nu=\mu, s=\frac{1}{2} r^{3} \ldots \ldots \ldots \ldots \ldots
$$


Accordingly these values give

$$
\begin{aligned}
\frac{\mu}{z}-\frac{1}{z^{\prime}}- & (\mu-1) r \\
= & \frac{\xi^{2}}{2}\left\{\mu\left(\frac{1}{z^{3}}-\frac{2 r}{z^{2}}+r^{3}\right)-\left(\frac{1}{z^{13}}-\frac{2 r}{z^{12}}+r^{3}\right)\right\} ;
\end{aligned}
$$

in the second member of which we have

$$
\begin{aligned}
& \mu\left(\frac{1}{z^{3}}-\frac{2 r}{z^{2}}+r^{3}\right)=\mu\left(\frac{1}{z}-r\right)\left(\frac{1}{z^{2}}-\frac{r}{z}-r^{2}\right), \\
& \frac{1}{z^{13}}-\frac{2 r}{z^{12}}+r^{3}=\left(\frac{1}{z^{\prime}}-r\right)\left(\frac{1}{z^{12}}-\frac{r}{z^{\prime}}-r^{2}\right),
\end{aligned}
$$

and may write

$$
\mu\left(\frac{1}{z}-r\right)=\frac{1}{z^{\prime}}-r=\frac{\mu}{\mu-1}\left(\frac{1}{z^{\prime}}-\frac{1}{z}\right) ;
$$

the formula for an ordinary spheric refraction is therefore thus found to be

$$
\left.\begin{array}{rl}
\frac{\mu}{z}-\frac{1}{z^{\prime}} & -(\mu-1) r \\
& =\frac{\mu}{\mu-1}\left(r-\frac{1}{z}-\frac{1}{z^{\prime}}\right)\left(\frac{1}{z}-\frac{1}{z^{\prime}}\right)^{2} \frac{\xi^{2}}{2},
\end{array}\right\} \ldots
$$

in which it may be remarked that $\left(\frac{1}{z}-\frac{1}{z^{\prime}}\right)^{2} \xi^{2}$ is the square of the angular deviation, and which is easily seen to agree with known results.

7. Returning to the crystal, let it be bounded by a second surface of revolution, infinitely near to the former, and about the same axis; and let the light emerge at this second surface into a vacuum again. The equation of the second surface being

$$
\zeta^{\prime}=\frac{1}{2} r^{\prime \prime} \xi^{\prime 2}+\frac{1}{4} s^{\prime} \xi^{\prime 4}, \ldots \ldots .
$$

and the ordinate of the intersection of the emergent ray with the axis being $z^{\prime \prime}$, the formula (24.) will apply to this new case by merely changing $r, s, z^{\prime}, z$ respectively to $-r^{\prime},-s^{\prime}$, $-z^{\prime \prime},-z$, without changing $\mu, \nu, \xi$; and we have

$$
\begin{aligned}
& \frac{-\nu^{2}}{\mu z}+\frac{1}{z^{\prime \prime}}+(\mu-1) r^{\prime} \\
& \left.\quad=\frac{\xi^{2}}{2}\left\{\frac{-v^{4}}{\mu^{3} z^{3}}+\frac{1}{z^{\prime \prime 3}}-2(\mu-1) s^{\prime}+2 r^{\prime}\left(\frac{v^{2}}{\mu z^{2}}-\frac{1}{z^{\prime \prime 2}}\right)\right\} \cdot\right\}
\end{aligned}
$$

And, adding the two equations (24.) and (28.), we find 
294 Sir W. R. Hamilton on a Lens of Uniaxal Crystal.

$$
\left.\begin{array}{rl}
\frac{1}{z^{\prime \prime}}- & \frac{1}{z^{\prime}}-(\mu-1)\left(r-r^{\prime}\right) \\
= & \frac{\xi^{2}}{2}\left\{\begin{array}{l}
\frac{1}{z^{\prime \prime 3}}-\frac{1}{z^{13}}+2(\mu-1)\left(s-s^{\prime}\right) \\
+\frac{2\left(r^{\prime}-r\right) y^{2}}{\mu z^{2}}-\frac{2 r^{\prime}}{z^{\prime \prime 2}}+\frac{2 r}{z^{1 / 2}}
\end{array}\right\}
\end{array}\right\} ;
$$

a formula for the focal lengths and aberrations of a lens of uniaxal crystal bounded by any two infinitely near surfaces, which have the optical axis of the crystal for their common axis of revolution : all cases of cusps or other singular curvatures at the common vertex being here set aside.

8. We shall content ourselves at present with drawing two conclusions from this formula. First, that because the extraordinary index $\nu$ disappears from the part unaffected with the small factor $\xi^{2}$, the central focus of the extraordinary rays, after emerging from the thin crystalline lens, coincides with the central focus of the ordinary rays which emerge from the same lens, wherever in the axis the focus of the incident rays may be: whereas Malus *, misled by an error of sign in a radical which he employed for expressing the law of extraordinary refraction in the case of an uniaxal crystal, thought that these foci, ordinary and extraordinary, might differ widely from each other. And second, that on account of the presence of $y$ in the term

$$
\frac{\left(r^{\prime}-r\right) v^{2} \xi^{2}}{\mu z^{2}}, \quad \ldots \ldots \ldots
$$

the ordinary and extraordinary aberrations cannot be exactly the same (the power $(\mu-1)\left(r-r^{\prime}\right)$ of the lens being supposed to be different from 0 ), unless the $z$ in the denominator of this term become infinite; that is, unless, wherever the focus of incident rays may be, the lens is so placed as to allow the rays within the crystal to be exactly parallel to the axis. If then a lens of this sort be used for the object-glass of a telescope, it seems to be desirable that its anterior surface, or that on which the parallel rays fall, should be plane, and that the correction of the aberration of figure should be effected entirely by another lens, composed of an uncrystallized material.

W. R. H.

Observatory of Trinity College, Dublin, September 14, 1841 .

* Perhaps it may not be improper to mention here, as I am not aware that the correction of Malus's result, respecting the extraordinary focal length of a lens of uniaxal crystal, has been hitherto published by any other person, that this correction occurred to me many years ago, in treating the question by my own methods, which made it scarcely possible to fall into the same error. 'The discrepance between my conclusion and his was, however, so great, that I was at first perplexed to account for it, until I traced that error of sign in his calculations, to which allusion has been made above. 